\title{
Efficient Pre-stressed Harmonic Analysis of RF-Microresonators by Means of Model Order Reduction
}

\author{
L. Del Tin ${ }^{1}$, R. Gaddi ${ }^{1}$, A.Gnudi ${ }^{1}$, E. Rudnyi ${ }^{2}$, A. Greiner ${ }^{2}$, J. G. Korvink ${ }^{2}$ \\ ${ }^{1}$ ARCES-DEIS, University of Bologna, Viale Risorgimento 2, 40136 Bologna, Italy. Email: 1deltin@arces.unibo.it \\ 2 IMTEK, Laboratory for simulation, Department of Microsystem Engineering, University of Freiburg, Georges-
} Koehler-Allee 103, 79110, Freiburg, Germany

\begin{abstract}
A simulation methodology to reduce computational time of pre-stressed harmonic analysis of radio frequency (RF) microresonators is demonstrated. The methodology is based on the application of model order reduction to a system of ordinary differential equations obtained after spatial discretization by finite element software. Model order reduction produces a low dimensional approximation of the original system and hence enables a substantial reduction of simulation time while maintaining a very small approximation error. The approach allows to perform rapid device design and optimization. Once the device design and working conditions have been defined its reduced model can also be used to implement a behavioural model that can be employed in system level simulations.
\end{abstract}

\section{Introduction}

Vibrating micromechanical structures with electrostatic actuation are good candidates for demanding frequency-selective applications, especially in the wireless telecommunication field. Recent years have therefore witnessed continuous efforts focused on their design and modeling. Good performance in terms of both quality factor and power consumption together with small dimensions, make micromechanical resonator a valid alternative to previously adopted devices, such as ceramic filters, SAW filters and quartz crystals. Moreover, RFMEMS devices offer the possibility for on-chip fabrication, which implies an overall cost reduction [1].

Microelectromechanical resonators are usually operated with a harmonic voltage of small amplitude, superimposed on a large bias voltage. The characterization of their frequency response requires therefore harmonic simulation in which deformation of the structure and nonlinear effects due to static loading are considered. This analysis is generally referred to as harmonic pre-stressed analysis. Pre-stress effects have significant influence on the total stiffness of the structure, and have therefore to be taken into account for correct harmonic simulation. For devices with simple geometries, analytical formulas are used to approximately compute the device resonance frequency and equivalent circuit models are used to simulate the frequency behaviour [2]. With the increase of geometrical complexity, the finite element method (FEM) is employed to improve modeling accuracy [3]. However, harmonic simulation of electromechanical problems using FEM is computationally expensive because two coupled energy domains are involved. FEM discretizes the device in space and leads to a system of ordinary differential equations (ODE) whose dimension is generally very large. Harmonic analysis requires the solution of such a system in the frequency domain for each frequency step. This is a major drawback when simulation is applied to device design optimization with respect to model parameters. Because of high computational complexity, finite element simulation in practice is often limited to a static or modal analysis.

In this paper, a simulation approach is presented that enables a significant speed-up of the harmonic prestressed analysis of electrostatically driven micromechanical devices. The approach is based on the application of model order reduction (MOR). Since harmonic analysis is a small signal linear analysis around a bias point, a technique for linear MOR can be applied. System matrices describing the electromechanical problem made by the FEM software ANSYS ${ }^{\circledR}$ are read directly from ANSYS binary files and a low-order model of the device is automatically extracted. This is done with the tool mor4ansys, which performs moment-matching model order reduction via the Arnoldi algorithm [4]. The reduced model, which takes into account both electrical and mechanical pre-stress effects, is then used to perform linear harmonic analysis. Simulation results for a lateral clamped-clamped beam resonator are presented and compared with the full model.

\section{Finite element modeling and simulation}

The finite element tool ANSYS is used for modeling of the microelectromechanical device. During the analysis electrical and mechanical domains are directly coupled. For this purpose, electromechanical coupling is modeled using transducer elements (TRANS126) implemented in ANSYS. These lumped elements model the capacitive response of a device to a motion in one direction. Each element has two nodes, with an electrical and a mechanical d.o.f. for each node, representing voltage and displacement along one of the coordinate directions. The electrostatic behaviour of the electromechanical device is then completely described in terms of capacitance between the conductive parts that are connected by nodes of the TRANS126 element. Therefore, only movable structures have to be represented and meshed. The relation between the capacitance and the distance (in the chosen direction) for each element can be computed automatically by ANSYS using the analytical formula for a parallel plate capacitor. Alternatively the relation can be specified on the basis of a separate series of electrostatic simulations for various distance values. This second approach allows to achieve a good degree of approximation for devices with complex geometry [5]. Electrostatic forces are then computed using energy principles. TRANS126 modeling capabilities are limited to devices having electrostatic forces between conductors 


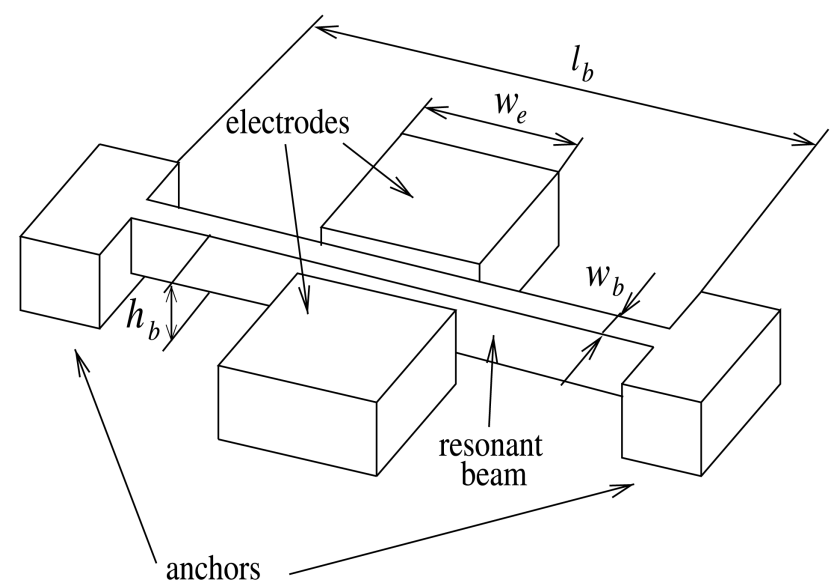

Figure 1: Schematic model of a two-port clampedclamped lateral beam resonator.

that can be accurately described as functions of a unidirectional displacement. This is the case for most of the micromachined mechanical resonators, for which it is generally possible to choose a preferential direction of movement.

After the model is built and meshed, the bias voltage is applied to the device and a static nonlinear simulation is performed. Uniform initial stresses for all materials present in the device can also be included in the model in order to take into account fabrication process induced strains.

During the nonlinear static analysis the stress stiffening matrix is obtained. This matrix describe the variation in mechanical stiffness of the structure due to the state of stress which results from its bending. The final system has the following form:

$$
\mathrm{M} \ddot{\boldsymbol{x}}+\mathrm{E} \dot{\boldsymbol{x}}+(\mathrm{K}+\mathrm{S}) \boldsymbol{x}=\mathbf{F}
$$

where $\mathrm{M}$ is the mass matrix, $\mathrm{E}$ the damping matrix, $\mathrm{K}$ the stiffness matrix, $\mathrm{S}$ the stress stiffening matrix and $\mathrm{F}$ the load vector for small signal harmonic analysis. System matrices and boundary conditions are stored in Ansys binary files. The matrix $\mathrm{K}$, nonlinear due to the presence of electrostatic elements, is evaluated at the bias point The sum of stiffening and stress stiffening matrices gives the total stiffness of the structure $\mathrm{K}^{\mathrm{tot}}$ that will be used for the harmonic analysis. Thus both mechanical and electrical effects of the applied bias voltage are taken into account. Since both terms of the total stiffness matrix are dependent from the bias voltage, matrices computation requires a nonlinear analysis for each bias voltage of interest. Each low-order model will then be extracted for a particular bias voltage.

\section{Model order reduction}

The current version of mor4ansys (1.8) does not allow to work with the stress stiffening matrix. Therefore the special command line tool dumpmatrices has been developed to extract the system (1). The program reads the system matrices directly from Ansys binary files after the nonlinear static analysis. Stiffness and stress stiffening matrices are stored separately and subsequently their sum is computed.
Table 1.: Material properties and dimensions used for the example considered.

\begin{tabular}{|c|c|}
\hline \multicolumn{2}{|c|}{ Material properties } \\
\hline Youngh modulus $[\mathrm{GPa}]$ & 167 \\
\hline Poisson ratio & 0.25 \\
\hline Density $\left[\mathrm{Kg} / \mathrm{m}^{3}\right]$ & 2330 \\
\hline \multicolumn{2}{|c|}{ Geometrical dimensions } \\
\hline Beam length $l_{b}[\mu \mathrm{m}]$ & 310 \\
\hline Beam width $w_{b}[\mu \mathrm{m}]$ & 6 \\
\hline Beam height $h_{b}[\mu \mathrm{m}]$ & 10 \\
\hline Electrode width $w_{e}[\mu \mathrm{m}]$ & 40 \\
\hline Gap $[\mathrm{nm}]$ & 600 \\
\hline
\end{tabular}

After this preparation step, the resulting system is reduced by mortansys. The model order reduction tool computes a low dimensional Krylov subspace by means of the Arnoldi algorithm, and projects the original system on this subspace. It has been proved that the obtained reduced system is a Padé-type approximant of the original system. This means that the reduced system matches the first $n$ moments in the Taylor expansion of the original transfer function, provided that $n$ is the dimension chosen for the reduced system. The algorithm is iterative by nature. This implies that any system of a lower order $r<n$ can be obtained by taking the first $r$ columns and rows of matrices in the reduced system.

After the reduced order system is derived, harmonic simulation is performed in Mathematica ${ }^{\circledR}$. Damping can be introduced in the model as mode preserving Rayleigh damping, or as a constant damping ratio. In the first case, the damping matrix $\mathrm{E}$ for the system is computed as a linear combination of the stiffness and mass matrices:

$$
\mathrm{E}=\alpha \mathrm{M}+\beta \mathrm{K}^{\mathrm{tot}}
$$

where $\alpha$ and $\beta$ are the two constant coefficients. In the second case, the damping matrix $\mathrm{C}$ is assumed to be proportional to the stiffness matrix only, with a proportion coefficient, which depends on frequency according to the relation:

$$
\beta_{c}=\frac{1}{\pi f} \xi
$$

where $\xi$ is the constant damping ratio. The following complex system of equations is then solved for every frequency of interest:

$$
\left(\mathrm{K}-\omega^{2} \mathrm{M}+j \omega \mathrm{E}\right)\left(x_{r}+j x_{i}\right)=\mathrm{F}_{r}+j \mathrm{~F}_{i}
$$

where $\omega=2 \pi f$ and the subscript $r$ and $i$ indicate respectively real and imaginary part of the considered quantities.

\section{Results}

The described approach has been applied to the harmonic pre-stressed simulation of a clamped-clamped beam two-port lateral microresonator. The device, shown in figure 1, presents a beam anchored at both ends whose movement is exited by one of the two side electrodes, while the other one is used for sensing. Dimension and 
material properties used in the simulation were taken from [6] and are summarized in table 1. According to these data, the estimated first resonance frequency of the device should be around $550 \mathrm{kHz}$. The reported quality factor $\mathrm{Q}$ of the device was used to choose a constant damping ratio as follows:

$$
\xi=\frac{1}{2 \mathrm{Q}}
$$

A 3D finite element model of the resonant beam and anchors have been created and meshed with hexahedral linear elements (SOLID45). The symmetry of the device with respect to its length and width axes has been exploited to model only one quarter of the structure. The electrostatic excitation has been described with 24 trans 126 elements connecting nodes on the lateral surface of the beam that faces the electrode and nodes created on the surface of the fixed electrode, which is not included otherwise in the solid model. The capacitance of each element is computed considering the SOLID45 element area while assuming a parallel plate capacitor condition.

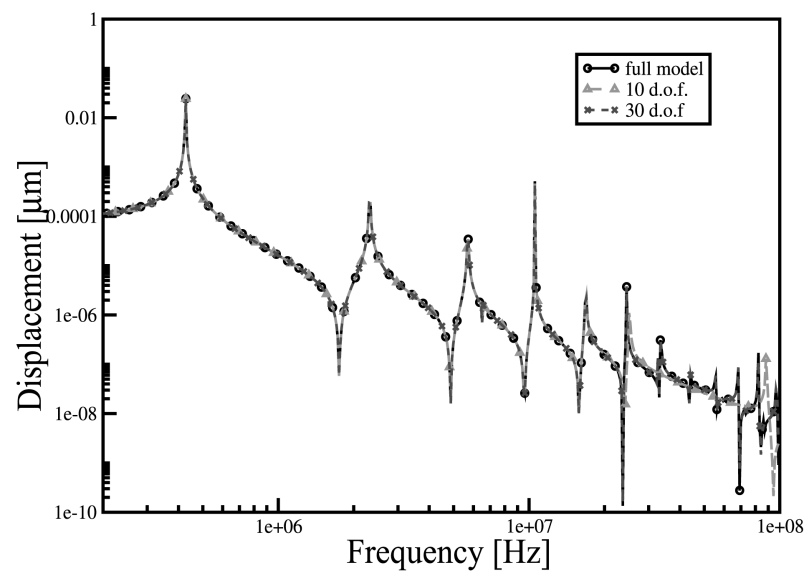

a.

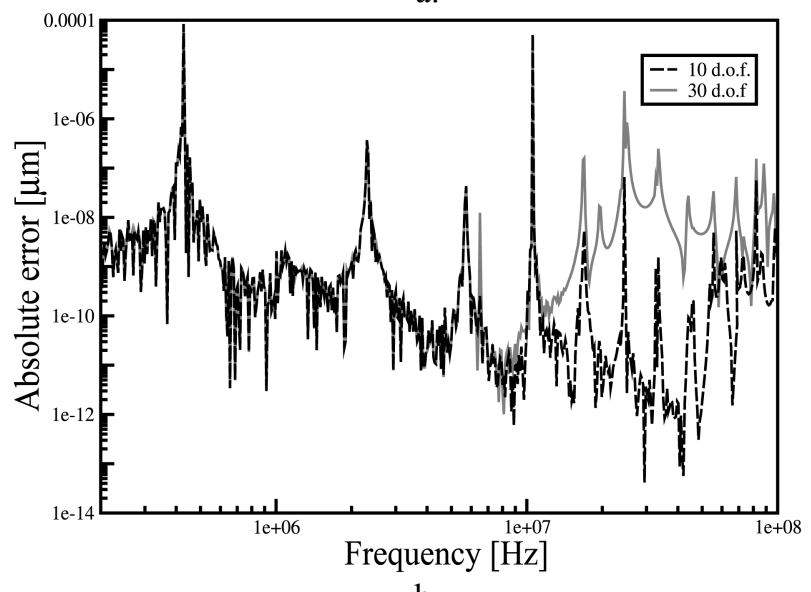

b.

Figure 2: a. Amplitude of the lateral displacement of the central part of the beam from the static position as a function of the frequency of the applied sinusoidal voltage. Simulation results obtained with the full model are compared with the ones obtained with the two reduced models(10 and 30 d.o.f). Graph b. shows the absolute error committed in both cases.
The device has been first polarized with a bias voltage of $9 \mathrm{~V}$ onto which a $0.1 \mathrm{~V}$ amplitude harmonic signal has been superimposed. Results obtained with the full system simulation were compared with the one obtained with different reduced order models. Figure $2 \mathrm{a}$ shows the amplitude of the lateral displacement at the center of the beam for the full model, having 3316 d.o.f., a 10 d.o.f. reduced model and a 30 d.o.f. reduced model, while figure $2 \mathrm{~b}$ shows the difference between the displacement of the full model and the reduced models. It can be noticed that both reduced models accurately describe the device behaviour in a wide range of the spectrum, so that the three curves can be hardly distinguished. In particular, the positions of the resonance peaks are correctly predicted, while a small error is present in the peak values. Ten d.o.f. are already sufficient to reproduce the first 5 resonance modes of the device with a relative error smaller than $1 \%$. For frequencies close to the operative frequency of the device, i.e. the first resonance frequency, the model can be reduced down to 5 d.o.f. while maintaining a good accuracy.

Figure 3 shows the different positions in frequency of the peak displacement amplitudes corresponding to different applied bias voltages. Results were obtained using 5 d.o.f. models computed at the three different voltages and show excellent agreement with the full model. The geometrical dimensions and material properties of the device render the stiffening of the structure caused by its static deformation negligible at the considered voltages. Therefore, due to the nonlinearity of the electrostatic force, an increase in the applied bias voltage causes the device resonance frequency to shift towards a lower value (spring softening). Since the electrostatic force is proportional to the bias voltage, the pick amplitude increases linearly with it. The pre-stress effects that influence the frequency behaviour of the device are therefore correctly taken into account in the simulation.

To evaluate the speed up achieved with the application of MOR, the time required by ANSYS to perform the harmonic pre-stressed analysis of the full model of the device was compared with the total time required by the

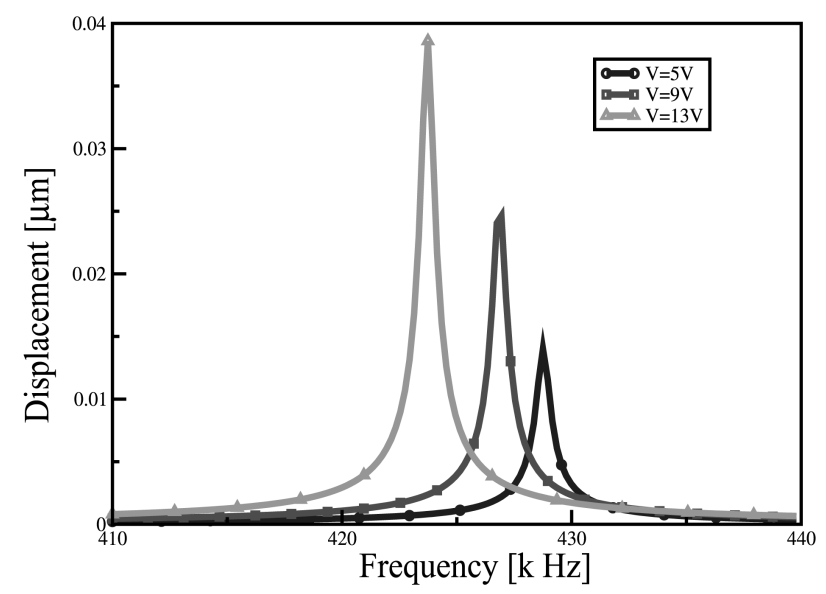

Figure 3: Frequency shift of the peak displacement of the structure for different values of the applied bias voltage. 
Table 2.: Times required for the various steps of harmonic pre-stressed analysis. The two values reported for harmonic analysis with Mathematica refer to the 10 and the 30 d.o.f. reduced order model

\begin{tabular}{|l|l|c|}
\hline \multicolumn{1}{|c|}{ Programs } & \multicolumn{1}{|c|}{ Computation } & time \\
\hline Ansys & Harmonic pre-stressed analysis & $1860 \mathrm{~s}$ \\
\hline \multirow{2}{*}{ Ansys } & $\begin{array}{l}\text { Static non linear analysis and } \\
\text { matrices computation }\end{array}$ & $11 \mathrm{~s}$ \\
\hline dumpmatrices & matrices extraction & $24 \mathrm{~s}$ \\
\hline \multirow{2}{*}{ mor4ansys } & reading matrices & $23 \mathrm{~s}$ \\
\cline { 2 - 3 } & vectors computation & $1.13 \mathrm{~s}$ \\
\cline { 2 - 3 } & matrices projection and writing & $1.62 \mathrm{~s}$ \\
\hline mathematica & harmonic analysis & $44-97 \mathrm{~ms}$ \\
\hline \multicolumn{2}{|l}{ total computation time with MOR } & $60 \mathrm{~s}$ \\
\hline
\end{tabular}

proposed procedure. The latter includes time to compute matrices with ANSYS, extract and reduce them with mor4ansys and finally perform the harmonic analysis of the reduced system. Table 2 shows computational times for each of these steps and the full scale FEM analysis with ANSYS (row in gray). In the case considered the application of model order reduction speed-up the simulation by approximately a factor 30 . Moreover, it can be noticed that the computational time needed for the harmonic solution of the reduced system is negligible with respect to the other steps. While for Ansys the full model computational time grows approximately linearly with the number of computed frequency steps, for the presented technique it almost does not depend on the number of frequencies. This is very advantageous when fine frequency discretization is required, which is typical for very high $\mathrm{Q}$ devices such as microelectromechanical resonators.

\section{Conclusions}

A simulation approach has been described to perform a harmonic pre-stressed analysis of electrostatically driven RF microresonators by means of model order reduction. The application of MOR techniques to the treatment of electromechanical problems was demonstrated with a test device and the achieved results were presented. The simulation was approximately 30 times faster. This could be even further improved if the technique would be embedded directly in the finite element software thus reducing data transfer overhead. The substantial decrease of the simulation time enables the use of the described procedure to adjust the devices parameters in the design phase, according to the desired performance goals. A valuable tool can also be envisaged for the mixed-domain circuit and system level simulations of complex circuits including both MEMS and solid state components. Each derived low-order model is specific for the geometry and the bias voltage considered. Nevertheless it can be used in system level simulations in order to evaluate the influence of the device on the overall circuit and system performances, aiming to optimize system level design. MOR has therefore been proven effective for harmonic pre-stressed elecromechanical simulation and is certainly promising for rapid design and optimization, both at device and system level.

\section{Acknowledgments}

The authors are grateful to the Ministry of Education University and Research (MIUR) for funding as part of the FIRB project "Enabling technologies for Wireless Reconfigurable Terminals" (RBNE01F582), to the German Science Foundation (DFG) for funding as part of the project MST-Kompakt and the Federal Ministry of Education and Research(BMBF) for funding as part of the project SIMOD.

\section{References}

1. Nguyen, C. T. -C., "Vibrating RF MEMS for Next Generation Wireless Application”, Proc. IEEE 2004 Custom Integrated Circuits Conf.

2. Veijola T. et al, "Large-Displacement modelling and Simulation of Micromechanical electrostatically Driven Resonators Using the Harmonic Balance Method", Microwave Symposium Digest., 2000 IEEE MTT-S International, 11-16 June 2000 Page(s):99 - 102 vol.1.

3. Laminmaeki T. et al, "Electromachanical analysis of micromechanical SOI-fabricated RF resonators", Proc. International Conference on Modeling and Simulation of Microsystem, MSM 2000, pp. 217-220

4. E. B. Rudnyi, J. G. Korvink. Model Order Reduction for Large Scale Engineering Models Developed in ANSYS. Lecture Notes in Computer Science, v. 3732, pp. 349-356, 2005.

http://www.imtek.uni-freiburg.de/simulation/mor4ansys/

5. Gyimesi, M. and Ostergaard, D., "ElectroMechanical Capacitor Element for MEMS Analysis in ANSYS", Proc. of Modelling and Simulation of Microsystems Conference, Puerto Rico (1999), pp. 270.

6. P. Ruther, et al, "Characterization of micro electromecanical HF resonators fabricated using a SOI-based low temperature process", Proc. IEEE Sensors 2003 Conference, pp.820-824. 